\title{
Spectral singularities in a non-Hermitian Friedrichs-Fano-Anderson model
}

\author{
Stefano Longhi \\ Dipartimento di Fisica and Istituto di Fotonica e Nanotecnologie del CNR, \\ Politecnico di Milano, Piazza L. da Vinci 32, I-20133 Milan, Italy
}

\begin{abstract}
Spectral singularities are predicted to occur in a non-Hermitian extension of the Friedrichs-FanoAnderson model describing the decay of a discrete state $|a\rangle$ coupled to a continuum of modes. A physical realization of the model, based on electronic or photonic transport in a semi-infinite tightbinding lattice with an imaginary impurity site at the lattice boundary, is proposed. The occurrence of the spectral singularities is shown to correspond either to a diverging reflection probability (for an amplifying impurity) or to a vanishing reflection probability (for an absorbing impurity) from the lattice boundary. In the former case, the spectral singularity of the resolvent is also responsible for the non-decay of state $|a\rangle$ into the continuum, in spite of the absence of bound states.
\end{abstract}

PACS numbers: 73.23.Ad, 03.65.Nk, 73.23.-b, 42.25.Bs

\section{INTRODUCTION.}

Over the last decade, a great attention has been devoted to investigate the properties of non-Hermitian physical systems. In particular, it has been shown that the framework of quantum mechanics can be extended by relaxing the common constraint of Hermiticity for the underlying Hamiltonian $H$ (see, for instance, [1 3 3 ] and references therein), provided that $H$ has a real energy spectrum and is diagonalizable. In this case, after a proper change of the inner product (metric) of the Hilbert space, the non-Hermitian Hamiltonian $H$ may be used to define a unitary quantum system [3]. Examples of such systems include non-Hermitian Hamiltonians with parity-time $(\mathcal{P} \mathcal{T})$ symmetry [4], in which the reality of the energy spectrum (bound as well as radiation states) below a symmetry-breaking transition has been proved for several complex potentials [1]. Unfortunately, non-Hermitian Hamiltonians possessing a real-valued energy spectrum may fail to be diagonalizable because of the occurrence of exceptional points in the point spectrum [5, 6], or of spectral singularities in the continuous part of the energy spectrum [7-9]. Exceptional points refer to the coalescence of two or more discrete eigenvalues together with their eigenvectors; their physical relevance has been investigated in several works, and different physical realizations have been proposed and experimentally demonstrated [10]. On the other hand, spectral singularities refer to divergences of the resolvent operator $G(z)=(z-H)^{-1}$ belonging to the continuous spectrum of $H$, i.e. which do not correspond to square-integrable eigenfunctions. As opposed to exceptional points, spectral singularities have received less attention from physicists and have been mainly viewed as a curious mathematical property of certain non-Hermitian operators [9]. The physical meaning and relevance of spectral singularities have been highlighted solely quite recently, notably by A. Mostafazadeh[11] (see also [8, 9, 12, 13]) in the framework of wave scattering by complex potentials [14]. Mostafazadeh showed that spectral singularities of a non-Hermitian Hamiltonian with a complex potential correspond to divergences of reflection and transmission coefficients of scattered states, i.e. to resonances with vanishing spectral width. He also investigated in details the appearance of spectral singularities in an electromagnetic realization of a non-Hermitian Hamiltonian with $\mathcal{P} \mathcal{T}$ symmetry based on a waveguide filled by an atomic gas [11, 15], following an earlier proposal by Ruschhaupt and coworkers [16].

It is the aim of this work to investigate the onset of spectral singularities in a non-Hermitian extension of the famous Friedrichs-Fano-Anderson (FFA) model [1719], which generally describes the decay of a discrete state coupled to a continuum of modes. The FFA model is encountered in different areas of physics, ranging from atomic physics [20 22] to quantum electrodynamics [23, 24] and condensed-matter physics [25 29]. The FFA model has been studied in the quantum theory of non-integrable systems [30] and used to describe unstable quantum systems, quantum mechanical decay and quantum Zeno dynamics [31, 32]. Simple models of single-particle electronic or photonic transport in tightbinding lattices can be also described by means of FFA Hamiltonians [25 29, 33 38]. In this work it is shown in particular that spectral singularities can be observed in photonic or electronic transport in semi-infinite tightbinding lattices with an imaginary impurity site at the lattice boundary, leading to either a diverging or a vanishing wave reflection from the lattice boundary.

The paper is organized as follows. In Sec.II we introduce a non-Hermitian extension of the FFA model, and derive the conditions for the appearance of spectral singularities. In particular, it is shown by direct calculations that the appearance of divergences of the resolvent operator $G(z)$ on the branch cut correspond to the nondiagonalizability of the FFA Hamiltonian. In Sec.III we present an example of non-Hermitian FFA model showing spectral singularities, which provides a simple model of electron or photonic transport in a semi-infinite tightbinding lattice with a boundary impurity site. It is shown that spectral singularities in this model correspond to either a vanishing or a diverging reflection probability from the lattice boundary. Finally, in Sec.IV the main conclu- 
sions are outlined.

\section{SPECTRAL SINGULARITIES IN A NON-HERMITIAN FRIEDRICHS-FANO-ANDERSON MODEL}

\section{A. The model}

The standard FFA model describes the interaction of a discrete state $|a\rangle$, of energy $E_{a}$, with a continuous set of states $|k\rangle$ with energy $E(k)$ (see, for instance, [22, 24 26, 29, 36]). Here $|a\rangle$ and $|k\rangle$ represent a complete set of Dirac states in the Hilbert space which satisfy the orthonormal conditions $\langle a \mid a\rangle=1,\langle a \mid k\rangle=0$ and $\left\langle k^{\prime} \mid k\right\rangle=\delta\left(k-k^{\prime}\right)$. The Hamiltonian of the full system can be written as $H=H_{0}+V$, where

$$
H_{0}=E_{a}|a\rangle\left\langle a\left|+\int d k E(k)\right| k\right\rangle\langle k|
$$

is the Hamiltonian of the non-interacting discrete and continuous states, and

$$
V=\int d k\left[v(k)|a\rangle\left\langle k\left|+v^{*}(k)\right| k\right\rangle\langle a|\right]
$$

is the (self-adjoint) interaction term, described by the spectral coupling function $v(k)$. Typically, we assume that the energy $E(k)$ of continuous states spans the interval $E_{1}<E<E_{2}$ (eventually $E_{2}=\infty$ ), and $E(k)$ is a monotonic function (either increasing or decreasing) of $k$, i.e. we assume that there are not energy degeneracies of continuous states. The state vector of the system $|\psi\rangle$ evolves according to the Schrödinger equation (with $\hbar=1)$

$$
i \frac{\partial|\psi\rangle}{\partial t}=H|\psi\rangle .
$$

Note that, provided that the energies $E_{a}$ and $E(k)$ are real-valued, the Hamiltonian $H$ is Hermitian. The spectrum of $H$ can be determined by either analyzing the singularities of the resolvent $G(z)=(z-H)^{-1}$, or by projecting the eigenvalue equation $H|\psi\rangle=E|\psi\rangle$ on the basis $\{|a\rangle,|k\rangle\}$. As is well known, the continuous spectrum of $H$ is $E_{1}<E<E_{2}$, i.e. the same as that of $H_{0}$, whereas the point spectrum can be either empty or composed by a number of discrete eigenvalues, either outside or embedded into the continuous spectrum (see, for instance, [22, 39, 40]).

We now relax the Hermiticity condition of the FFA model by allowing the 'energy' $E_{a}$ of the discrete state $|a\rangle$ to be complex valued. However, we will assume that the spectrum of $H$ remains real-valued in spite of the nonHermiticity of $H$. The condition for the spectrum of $H$ to remain real-valued will be discussed below in Sec.II.B and corresponds to the absence of bound states, i.e. to an empty point spectrum. This means that a non-Hermitian
FFA Hamiltonian has a real-valued energy spectrum if and only if its spectrum is purely continuous. We are interested here to determine, if any, the appearance of spectral singularities of $H$, which would prevent $H$ to be diagonalizable. This problem can be addressed in two ways: (i) by the determination of the resolvent $G(z)$, and (ii) by a direct diagonalization of $H$ following the original procedure by Fano [18], extended to account for the non-Hermitian nature of $H$. In the former case, a spectral singularity at $\mathcal{E}=\mathcal{E}_{0}$, embedded in the continuous spectrum $\left(E_{1}, E_{2}\right)$, is revealed as a divergence of the Green function $\mathcal{G}(x, y ; z)=\langle x \mid G(z) y\rangle$ in a neighborhood of $z=\mathcal{E}_{0}$, divergence which does not corresponds to a bound state embedded in the continuum [9]. In the latter case, a spectral singularity at $\mathcal{E}=\mathcal{E}_{0}$ occurs when $\left\langle\mathcal{E}_{0}^{\dagger} \mid \mathcal{E}_{0}\right\rangle=0$, where $\left|\mathcal{E}_{0}\right\rangle$ and $\left|\mathcal{E}_{0}^{\dagger}\right\rangle$ are the eigenfunctions of $H$ and of its adjoint $H^{\dagger}$, respectively, corresponding to the eigenvalue $\mathcal{E}_{0}[9]$.

\section{B. Spectral singularities: the resolvent approach}

For a given complex number $z$, the resolvent operator $G(z)$ of the Hamiltonian $H$ is defined as

$$
G(z)=(z-H)^{-1},
$$

i.e. $G(z)(z-H)=(z-H) G(z)=\mathcal{I}$, where $\mathcal{I}$ is the identity operator. The knowledge of the resolvent of $H$, for any $z$, is equivalent to the knowledge of the set of eigenfunctions and eigenvalues of $H$. In particular, the singularities of $G(z)$ in the complex plane define the spectrum of $H$ : an eigenvalue $\mathcal{E}$ belonging to the point spectrum of $H$ is a pole of $G(z)$, whereas the branch cut of $G(z)$ determines the continuous part of the spectrum of $H$. The Hamiltonian $H$ is said to have a spectral singularity at $\mathcal{E}=\mathcal{E}_{0}$, where $\mathcal{E}_{0}$ belongs to the continuous spectrum of $H$, if the function

$$
\mathcal{G}_{\chi, \varphi}(z)=\langle\chi \mid G(z) \varphi\rangle
$$

is unbounded in the neighborhood of $z=\mathcal{E}_{0}$, and $\mathcal{E}_{0}$ does not belong to the point spectrum of $H$, i.e. it does not corresponds to a bound state embedded in the continuum [9]. In the previous equation, $|\chi\rangle$ and $|\varphi\rangle$ are two assigned functions of the Hilbert space; in particular, for $|\chi\rangle=|x\rangle$ and $|\varphi\rangle=|y\rangle$ one obtains the coordinate representation of the resolvent $G$, i.e. the Green function $\mathcal{G}(x, y ; z)=\langle x \mid G(z) y\rangle$. An interesting property of the FFA Hamiltonian is the possibility to calculate the resolvent in a closed form. The procedure to calculate $G(z)$ is well known for the Hermitian case (see, for instance, [26]), and can be extended mutatis mutandis to the non-Hermitian FFA model considered in this work. As detailed in the Appendix A, the matrix elements of the resolvent $G(z)$ on the complete basis $\{|a\rangle,|k\rangle\}$ read explicitly 


$$
\begin{aligned}
\mathcal{G}_{a, a} & \equiv\langle a \mid G(z) a\rangle=\frac{1}{z-E_{a}-\Sigma(z)} \\
\mathcal{G}_{a, k} & \equiv\langle a \mid G(z) k\rangle=\frac{v(k)}{(z-E(k))\left(z-E_{a}-\Sigma(z)\right)} \\
\mathcal{G}_{k, a} & \equiv\langle k \mid G(z) a\rangle=\frac{v^{*}(k)}{(z-E(k))\left(z-E_{a}-\Sigma(z)\right)} \\
\mathcal{G}_{k, k^{\prime}} & \equiv\left\langle k \mid G(z) k^{\prime}\right\rangle=\frac{v\left(k^{\prime}\right) v^{*}(k)}{(z-E(k))\left(z-E\left(k^{\prime}\right)\right)\left(z-E_{a}-\Sigma(z)\right)}+\frac{\delta\left(k-k^{\prime}\right)}{z-E\left(k^{\prime}\right)}
\end{aligned}
$$

where $\Sigma(z)$ is the self-energy, defined by

$$
\Sigma(z)=\int d k \frac{|v(k)|^{2}}{z-E(k)} .
$$

Note that, by introducing the density of states $\rho(E)=$ $(\partial E / \partial k)^{-1}$ and letting $V(E)=\rho(E)|v(E)|^{2}(V(E)=0$ for $E>E_{2}$ and $\left.E<E_{1}\right)$, the self-energy can be written in the equivalent form

$$
\Sigma(z)=\int_{E_{1}}^{E_{2}} d E \frac{V(E)}{z-E} .
$$

Note also that $\Sigma(z)$ is not defined on the segment $\left(E_{1}, E_{2}\right)$ of the real axis, and one has

$$
\Sigma\left(z=\mathcal{E} \pm i 0^{+}\right)=\Delta(\mathcal{E}) \mp i \pi V(\mathcal{E})
$$

where

$$
\Delta(\mathcal{E})=P \int_{E_{1}}^{E_{2}} d E \frac{V(E)}{\mathcal{E}-E}
$$

$P$ denotes the principal value, and $\mathcal{E}$ is real-valued. Therefore, $\Sigma(z)$ has a branch cut in the interval $\left(E_{1}, E_{2}\right)$.

For two assigned functions $|\chi\rangle=\chi_{a}|a\rangle+\int d k \chi(k)|k\rangle$ and $|\varphi\rangle=\varphi_{a}|a\rangle+\int d k \varphi(k)|k\rangle$ of the Hilbert space, the complex function $\mathcal{G}_{\chi, \varphi}(z)=\langle\chi \mid G(z) \varphi\rangle$ can be readily calculated as

$$
\mathcal{G}_{\chi, \varphi}(z)=\chi_{a}^{*} \varphi_{a} \mathcal{G}_{a, a}(z)+\chi_{a}^{*} \int d k \varphi(k) \mathcal{G}_{a, k}(z)+\varphi_{a} \int d k \chi^{*}(k) \mathcal{G}_{k, a}(z)+\int d k d k^{\prime} \chi^{*}\left(k^{\prime}\right) \varphi(k) \mathcal{G}_{k^{\prime}, k}(z)
$$

Substitution of Eqs.(6-9) into Eq.(14) finally yields

$$
\mathcal{G}_{\chi, \varphi}(z)=\mathcal{G}_{a, a}(z) \Phi_{1}(z)+\Phi_{2}(z)
$$

where we have set

$$
\begin{aligned}
\Phi_{1}(z) & =\chi_{a}^{*} \varphi_{a}+\chi_{a}^{*} \int d E \frac{\rho(E) \varphi(E) v(E)}{z-E}+\varphi_{a} \int d E \frac{\rho(E) \chi^{*}(E) v^{*}(E)}{z-E}+ \\
& +\left(\int d E \frac{\rho(E) v(E) \varphi(E)}{z-E}\right)\left(\int d E \frac{\rho(E) v^{*}(E) \chi^{*}(E)}{z-E}\right) \\
\Phi_{2}(z) & =\int d E \frac{\rho(E) \chi^{*}(E) \varphi(E)}{z-E} .
\end{aligned}
$$

We are now ready to determine the singularities of the resolvent, i.e. the spectrum of $H$ and possible spectral singularities. To this aim, let us notice that $\Phi_{1}(z)$ and $\Phi_{2}(z)$ are bounded functions of $z$ and have a branch cut 
on the segment $\left(E_{1}, E_{2}\right)$ of the real axis (as for the selfenergy $\Sigma$ ). According to Eq.(15), we may therefore limit to consider the singularities of $\mathcal{G}_{a, a}(z)$. From Eqs.(6), (11) and (12) one can conclude that:

(i) The continuous spectrum of $H$ is the same as that of $H_{0}$, i.e. the interval $\left(E_{1}, E_{2}\right)$ of the real axis, where $G(z)$ has a branch cut.

(ii) The point spectrum of $H$, corresponding to bound states outside the continuum, are the complex roots $z$ of the equation

$$
z-E_{a}=\Sigma(z)
$$

in correspondence of which the resolvent $G(z)$ has a pole. (iii) A spectral singularity $\mathcal{E}_{0}$ of the continuous $\operatorname{spectrum}\left(E_{1}, E_{2}\right)$ is any solution of the coupled equations

$$
\begin{aligned}
\operatorname{Im}\left(E_{a}\right) & = \pm \pi V\left(\mathcal{E}_{0}\right) \\
\mathcal{E}_{0}-\operatorname{Re}\left(E_{a}\right) & =\Delta\left(\mathcal{E}_{0}\right)
\end{aligned}
$$

provided that $\operatorname{Im}\left(E_{a}\right) \neq 0[41]$.

Note that real-valued energies can not belong to the point spectrum of $H$ because Eq.(18) does not have real-valued roots whenever $H$ is non-Hermitian [42]. This means that the point spectrum of $H$, if not empty, is strictly complexvalued. On the other hand, the continuous part of the spectrum is real-valued according to the property (i). Therefore, we may conclude that the energy spectrum of the non-Hermitian FFA Hamiltonian is real-valued if and only if its spectrum is purely continuous. In this case, spectral singularities in the continuous spectrum occur whenever Eqs.(19) and (20) can be simultaneously satisfied. It should be noted that the behavior of $G(z)$ in the neighborhood of the spectral singularity $z=\mathcal{E}_{0}$ on the continuous spectrum is different for an 'absorbing' $\left[\operatorname{Im}\left(E_{a}\right)<0\right]$ and for an 'amplifying' $\left[\operatorname{Im}\left(E_{a}\right)>0\right]$ complex energy $E_{a}$ of state $|a\rangle$. Since the spectral coupling $V\left(\mathcal{E}_{0}\right)$ is always positive, for an absorbing complex energy Eq.(19) can be satisfied by taking the lower (negative) sign on the right hand side; correspondingly, from Eqs.(6) and (12) it follows that $\mathcal{G}_{a, a}(z)$ is unbounded when $z \rightarrow \mathcal{E}_{0}$ from the bottom of the real energy axis, i.e. for $\operatorname{Im}(z)<0$, but $\mathcal{G}_{a, a}(z)$ remains bounded when $z \rightarrow \mathcal{E}_{0}$ with $\operatorname{Im}(z)>0$ [43]. Conversely, for an 'amplifying' complex energy $\operatorname{Im}\left(E_{a}\right)>0$, Eq.(19) can be satisfied by taking the upper (positive) sign on the right hand side; correspondingly, $\mathcal{G}_{a, a}(z)$ is unbounded when $z \rightarrow \mathcal{E}_{0}$ with $\operatorname{Im}(z)>0$, but it remains bounded when $z \rightarrow \mathcal{E}_{0}$ with $\operatorname{Im}(z)<0$ [43]. Such a different behavior of spectral singularities for an absorbing or an amplifying complex energy $E_{a}$ has some relevant physical implications, that will be discussed in Sec.II.D and Sec.III.C. In Sec.II.D it will be shown that the appearance of a spectral singularity in the amplifying case is responsible for the non-decay of state $|a\rangle$ into the continuum, in spite of the absence of bound states; in Sec.III.C the interplay between spectral singularities and wave scattering will be investigated for a semi-infinite tight-binding lattice realization of the FFA Hamiltonian.

\section{Spectral singularities: the Fano diagonalization procedure}

Let us assume that the spectrum of the non-Hermitian Hamiltonian $H=H_{0}+V$ is real-valued. As shown in the previous subsection, this implies that the spectrum of $H$ is purely continuous and spans the interval $\left(E_{1}, E_{2}\right)$. Let us indicate by $|\mathcal{E}\rangle$ the (improper) eigenfunction of $H$ corresponding to the eigenvalue $\mathcal{E}$, and by $\left|\mathcal{E}^{\dagger}\right\rangle$ the (improper) eigenfunction of the adjoint $H^{\dagger}$ corresponding to the same eigenvalue $\mathcal{E}$. For $H$ to be diagonalizable, the set of functions $\left\{|\mathcal{E}\rangle,\left|\mathcal{E}^{\dagger}\right\rangle\right\}$ must form a complete biorthonormal basis of Hilbert space [9], that is

$$
\left\langle\mathcal{E}^{\dagger} \mid \mathcal{E}^{\prime}\right\rangle=\delta\left(\mathcal{E}-\mathcal{E}^{\prime}\right), \quad \int_{E_{1}}^{E_{2}} d \mathcal{E}|\mathcal{E}\rangle\left\langle\mathcal{E}^{\dagger}\right|=\mathcal{I}
$$

A spectral singularity at $\mathcal{E}=\mathcal{E}_{0}$ sets in when

$$
\left\langle\mathcal{E}_{0} \mid \mathcal{E}_{0}^{\dagger}\right\rangle=0
$$

which prevents $H$ to be diagonalizable. To determine the onset of spectral singularities of the FFA Hamiltonian $H$, we can thus proceed by calculating the eigenfunctions $|\mathcal{E}\rangle$ of $H$ and $\left|\mathcal{E}^{\dagger}\right\rangle$ of its adjoint $H^{\dagger}$ following the diagonalization procedure used by Fano [18] in the problem of atomic autoionizing resonances (see also [17, 21]), properly modified to account for the non-Hermitian nature of $H$. To this aim, we expand the eigenstates $|\mathcal{E}\rangle$ and $\left|\mathcal{E}^{\dagger}\right\rangle$ on the orthonormal and complete basis $\{|a\rangle,|k\rangle\}$ as

$$
\begin{aligned}
|\mathcal{E}\rangle & =\alpha(\mathcal{E})|a\rangle+\int d k \beta(\mathcal{E}, k)|k\rangle \\
\left|\mathcal{E}^{\dagger}\right\rangle & =\alpha^{\dagger}(\mathcal{E})|a\rangle+\int d k \beta^{\dagger}(\mathcal{E}, k)|k\rangle
\end{aligned}
$$

with expansion coefficients $\alpha(\mathcal{E}), \alpha^{\dagger}(\mathcal{E}), \quad \beta(\mathcal{E}, k)$ and $\beta^{\dagger}(\mathcal{E}, k)$ to be determined. Note that

$$
\left\langle\mathcal{E}^{\prime} \mid \mathcal{E}^{\dagger}\right\rangle=\alpha^{*}\left(\mathcal{E}^{\prime}\right) \alpha^{\dagger}(\mathcal{E})+\int d k \beta^{*}\left(\mathcal{E}^{\prime}, k\right) \beta^{\dagger}(\mathcal{E}, k)
$$

Using Eqs.(1), (2) and (23), the eigenvalue equation $H|\mathcal{E}\rangle=\mathcal{E}|\mathcal{E}\rangle$ yields the following coupled equations for the expansion coefficients $\alpha(\mathcal{E})$ and $\beta(\mathcal{E}, k)$ :

$$
\begin{gathered}
\left(E_{a}-\mathcal{E}\right) \alpha(\mathcal{E})+\int d k v(k) \beta(\mathcal{E}, k)=0 \\
{[E(k)-\mathcal{E}] \beta(\mathcal{E}, k)+v^{*}(k) \alpha(\mathcal{E})=0 .}
\end{gathered}
$$

Equation $(27)$ can be solved for $\beta(\mathcal{E}, k)$ and substituted into Eq. (26). However, there is a singularity at $E(k)=\mathcal{E}$, so that $1 /[E(k)-\mathcal{E}]$ must be written as its principal and delta function parts, yielding (see [18, 21])

$\beta(\mathcal{E}, k)=-v^{*}(k) \alpha(\mathcal{E})\left(\mathrm{P} \frac{1}{E(k)-\mathcal{E}}+z(\mathcal{E}) \delta(E(k)-\mathcal{E})\right)$.

The coefficient $z(\mathcal{E})$ in front of the delta function on the right hand side of Eq.(28) is determined by substituting 
Eq.(28) into Eq.(26) and requiring that $\alpha(\mathcal{E})$ does not vanish. This yields

$$
z(\mathcal{E})=\frac{E_{a}-\mathcal{E}+\Delta(\mathcal{E})}{V(\mathcal{E})}
$$

where $\Delta(\mathcal{E})$ is defined by Eq. $(13), V(\mathcal{E})=\rho(\mathcal{E})|v(\mathcal{E})|^{2}$ and $\rho(\mathcal{E})=(\partial E(k) / \partial k)^{-1}$ is the density of states.

Similarly, the eigenvalue equation $H^{\dagger}\left|\mathcal{E}^{\dagger}\right\rangle=\mathcal{E}\left|\mathcal{E}^{\dagger}\right\rangle$ yields

$$
\begin{aligned}
\left(E_{a}^{*}-\mathcal{E}\right) \alpha^{\dagger}(\mathcal{E})+\int d k v(k) \beta^{\dagger}(\mathcal{E}, k) & =0 \\
{[E(k)-\mathcal{E}] \beta^{\dagger}(\mathcal{E}, k)+v^{*}(k) \alpha^{\dagger}(\mathcal{E}) } & =0
\end{aligned}
$$

and the following expression of the coefficient $\beta^{\dagger}(\mathcal{E}, k)$ can be derived following the same procedure as above

$\beta^{\dagger}(\mathcal{E}, k)=-v^{*}(k) \alpha^{\dagger}(\mathcal{E})\left(\mathrm{P} \frac{1}{E(k)-\mathcal{E}}+z^{*}(\mathcal{E}) \delta(E(k)-\mathcal{E})\right)$.

Substitution of Eqs.(28) and (32) into Eq.(25) yields

$$
\left\langle\mathcal{E}^{\prime} \mid \mathcal{E}^{\dagger}\right\rangle=\alpha^{*}\left(\mathcal{E}^{\prime}\right) \alpha^{\dagger}(\mathcal{E}) F\left(\mathcal{E}, \mathcal{E}^{\prime}\right)
$$

where we have set

$$
\begin{aligned}
F\left(\mathcal{E}, \mathcal{E}^{\prime}\right) & =\int_{E_{1}}^{E_{2}} d E V(E)\left(\mathrm{P} \frac{1}{E-\mathcal{E}}+z^{*}(\mathcal{E}) \delta(E-\mathcal{E})\right) \\
& \times\left(\mathrm{P} \frac{1}{E-\mathcal{E}^{\prime}}+z^{*}\left(\mathcal{E}^{\prime}\right) \delta\left(E-\mathcal{E}^{\prime}\right)\right)+1
\end{aligned}
$$

The calculation of the integral on the right hand side of Eq.(34) is complicated by the product of two principal parts, which must be properly resolved into its partialfraction and singular terms (see [18] or the Appendix of Ref.[21]). Taking into account that

$$
\begin{aligned}
\mathrm{P} \frac{1}{E-\mathcal{E}} \mathrm{P} \frac{1}{E-\mathcal{E}^{\prime}} & =\mathrm{P} \frac{1}{\mathcal{E}-\mathcal{E}^{\prime}}\left(\mathrm{P} \frac{1}{\mathcal{E}^{\prime}-E}-\mathrm{P} \frac{1}{\mathcal{E}-E}\right)+ \\
& +\pi^{2} \delta\left(\mathcal{E}^{\prime}-E\right) \delta(\mathcal{E}-E)
\end{aligned}
$$

and using Eq.(29), from Eq.(34) one obtains

$$
F\left(\mathcal{E}, \mathcal{E}^{\prime}\right)=V(\mathcal{E})\left[\pi^{2}+z^{* 2}(\mathcal{E})\right] \delta\left(\mathcal{E}-\mathcal{E}^{\prime}\right)
$$

so that [see Eq.(33)]

$$
\left\langle\mathcal{E}^{\prime} \mid \mathcal{E}^{\dagger}\right\rangle=\alpha^{*}(\mathcal{E}) \alpha^{\dagger}(\mathcal{E}) V(\mathcal{E})\left[\pi^{2}+z^{* 2}(\mathcal{E})\right] \delta\left(\mathcal{E}-\mathcal{E}^{\prime}\right) .
$$

In order $\left\{|\mathcal{E}\rangle,\left|\mathcal{E}^{\dagger}\right\rangle\right\}$ to represent a complete biorthonormal set of functions [Eq.(21)], the amplitudes $\alpha(\mathcal{E})$ and $\alpha^{\dagger}(\mathcal{E})$ should be thus normalized such that

$$
\alpha^{*}(\mathcal{E}) \alpha^{\dagger}(\mathcal{E}) V(\mathcal{E})\left[\pi^{2}+z^{* 2}(\mathcal{E})\right]=1 .
$$

For the Hermitian FFA model, the energy $E_{a}$ is realvalued, $z(\mathcal{E})$ turns out to be real-valued and $\alpha^{\dagger}(\mathcal{E})=$ $\alpha(\mathcal{E})$ [because of $\left|\mathcal{E}^{\dagger}\right\rangle=|\mathcal{E}\rangle$ ], so that Eq.(38) is solved to yield

$$
|\alpha(\mathcal{E})|^{2}=\frac{V(\mathcal{E})}{\pi^{2} V^{2}(\mathcal{E})+\left[E_{a}-\mathcal{E}+\Delta(\mathcal{E})\right]^{2}}
$$

according to Fano [18]. The physical meaning of Eq.(39) is well known [18]: owing to the coupling with the continuum, the discrete state $|a\rangle$ is 'diluited' throughout a set of continuous states (i.e., it becomes a resonance for $H$ ) with a typical resonance curve $|\alpha(\mathcal{E})|^{2}$ peaked at $\mathcal{E} \simeq E_{a}-\Delta\left(E_{a}\right)$ of width $\simeq \pi V\left(E_{a}\right)$ [4].

For the non-Hermitian FFA model, the energy $E_{a}$ is complex-valued and from Eq.(37) it follows that a spectral singularity can appear at the energy $\mathcal{E}=\mathcal{E}_{0}$ of the continuous spectrum such that $\pi^{2}+z^{* 2}\left(\mathcal{E}_{0}\right)=0$, i.e. when

$$
z^{*}\left(\mathcal{E}_{0}\right)=\mp i \pi .
$$

Using Eq.(29), Eq.(40) yields the following conditions for the appearance of a spectral singularity in the nonHermitian FFA model

$$
\operatorname{Im}\left(E_{a}\right)= \pm \pi V\left(\mathcal{E}_{0}\right), \operatorname{Re}\left(E_{a}\right)-\mathcal{E}_{0}+\Delta\left(\mathcal{E}_{0}\right)=0
$$

which are precisely Eqs.(19) and (20) derived in Sec.II.B following the resolvent approach to spectral singularities.

\section{Spectral singularities and decay dynamics}

Hermitian FFA models are often used to describe the evolution of unstable quantum systems and the related problem of quantum mechanical decay and deviations from an exponential decay law (see, for instance, [31, 32. and references therein). If the system is initially prepared in state $|a\rangle$, i.e. if $|\psi(t=0)\rangle=|a\rangle$, the survival probability $P(t)$ to find the system at state $|a\rangle$ at a successive time $t$ is given by $P(t)=\left|c_{a}(t)\right|^{2}$, where

$$
c_{a}(t)=\langle a \mid \psi(t)\rangle=\langle a|\exp (-i H t)| a\rangle .
$$

Here we consider the case of a non-Hermitian FFA Hamiltonian $H$ with a real-valued (and thus purely continuous) energy spectrum, and briefly discuss the physical implications of spectral singularities of $H$ on the decay dynamics of survival probability $P(t)$. The temporal evolution operator $\exp (-i H t)$ of the system can be written in terms of the resolvent $G(z)$ as

$$
\exp (-i H t)=\frac{i}{2 \pi} \int_{\mathrm{B}} d z G(z) \exp (-i z t)
$$

where the Bromwich path $\mathrm{B}$ is any horizontal line $\operatorname{Im}(z)=$ const $>0$ in the upper half of the complex $z$ plane [see Fig.1(a)]. Substitution of Eq.(43) into Eq.(42) yields

$$
c_{a}(t)=\frac{i}{2 \pi} \int_{\mathrm{B}} d z \mathcal{G}_{a, a}(z) \exp (-i z t)
$$

where the matrix element $\mathcal{G}_{a, a}(z)$ of the resolvent is given by Eq.(6). As discussed in Sec.II.B, $\mathcal{G}_{a, a}(z)$ is analytic in the full complex plane, expect for the branch cut on the real axis, from $z=E_{1}$ to $z=E_{2}$ (see Fig.1), where it 

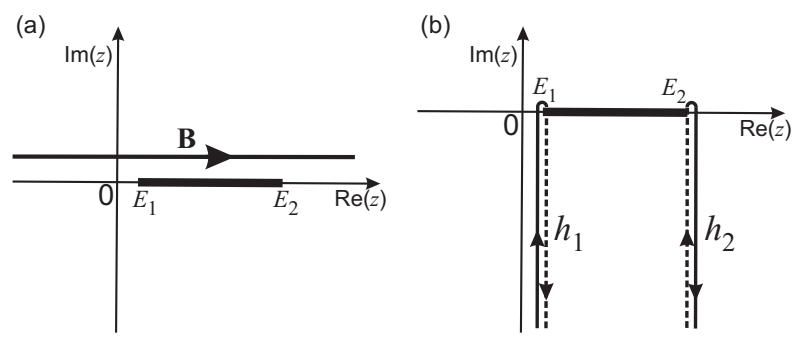

FIG. 1: (a) Integration contour (Bromwich path B) in the $\operatorname{Im}(z)>0$ complex plain entering in Eqs.(43) and (44). The bold horizontal segment on the real axis is the continuous spectrum of $H$ and corresponds to a branch cut of $\mathcal{G}_{a, a}(z)$. (b) Integration contour (Hankel paths $h_{1}$ and $h_{2}$ ) after deformation of the Bromwich path. The integration along the solid (dashed) curves of the Hankel paths is made on the first (second) Riemannian sheet of $\mathcal{G}_{a, a}(z)$.

can also become unbounded at energies $\mathcal{E}_{0}$ corresponding to spectral singularities. The integral on the right hand side of Eq.(44) can be evaluated by the residue method after suitably closing the Bromwich path $\mathrm{B}$ with a contour in the $\operatorname{Im}(z)<0$ half-plane (see, e.g., 31]). Since the closure crosses the branch cut on the real axis, the contour must necessarily pass into the second Riemannian sheet in the sector of the $\operatorname{Im}(z)<0$ half-plane with $E_{1}<\operatorname{Re}(z)<E_{2}$, whereas it remains in the first Riemannian sheet in the other two sectors $\operatorname{Re}(z)<E_{1}$ and $\operatorname{Re}(z)>E_{2}$ of the $\operatorname{Im}(z)<0$ half-plane. To properly close the contour, it is thus necessary to go back and turn around the two branch points of the cut at $z=E_{1}$ and $z=E_{2}$, following the Hankel paths $h_{1}$ and $h_{2}$ as shown in Fig.1(b). Indicating by $\mathcal{G}_{a, a}^{(I I)}(z)$ the analytic continuation of $\mathcal{G}_{a, a}(z)$ from the upper $[\operatorname{Im}(z)>0]$ to the lower $[\operatorname{Im}(z)<0]$ half-plane across the branch cut, and by $z_{1}, z_{2}, \ldots$ the (possible) poles of $\mathcal{G}_{a, a}^{(I I)}(z)$ that lies in the sector $\operatorname{Im}(z)<\eta, E_{1}<\operatorname{Re}(z)<E_{2}$ of the complex plane (with $\eta>0$ arbitrarily small), assuming that the poles are of first order one can write

$$
c_{a}(t)=\sum_{z_{k}} R_{k} \exp \left(-i z_{k} t\right)+\mathcal{C}(t)
$$

where $R_{k}$ is the residue of $\mathcal{G}_{a, a}^{(I I)}(z)$ at $z=z_{k}$, and $\mathcal{C}(t)$ is the contribution from the contour integration along the Hankel paths $h_{1}$ and $h_{2}$

$$
\begin{aligned}
\mathcal{C}(t) & =\frac{i}{2 \pi} \int_{E_{1}-i \infty}^{E_{1}+i 0} d z\left[\mathcal{G}_{a, a}(z)-\mathcal{G}_{a, a}^{(I I)}(z)\right] \exp (-i z t)+ \\
& +\frac{i}{2 \pi} \int_{E_{2}-i \infty}^{E_{2}+i 0} d z\left[\mathcal{G}_{a, a}^{(I I)}(z)-\mathcal{G}_{a, a}(z)\right] \exp (-i z t)(46)
\end{aligned}
$$

The cut contribution $\mathcal{C}(t)$ vanishes as $t \rightarrow \infty$, however it is responsible for the appearance of nonexponential features in the decay dynamics 31]. For the Hermitian FFA model, the poles $z_{k}$ of $\mathcal{G}_{a, a}^{(I I)}(z)$ lie below the real axis, i.e. $\operatorname{Im}\left(z_{k}\right)<0$, because $\mathcal{G}_{a, a}\left(z=E+i 0^{+}\right)$is a bounded function. Therefore $P(t) \rightarrow 0$ as $t \rightarrow \infty$ [45]. Typically, $\mathcal{G}_{a, a}^{(I I)}(z)$ has one pole below the imaginary axis [31], so that the decay of $c_{a}(t)$ follows an exponential law (with decay rate determined by the imaginary part of the pole), corrected by the cut contribution $\mathcal{C}(t)$. Let us consider now the non-Hermitian FFA model with a spectral singularity at $\mathcal{E}=\mathcal{E}_{0}$ in the continuous spectrum. In this case, we have to distinguish two cases. For an absorbing complex energy $E_{a}$, as shown in Sec.II.B $\mathcal{G}_{a, a}(z)$ is bounded for $z=E+i 0^{+}$; therefore, its analytic continuation $\mathcal{G}_{a, a}^{(I I)}(z)$ in the lower-half complex plane is bounded for $z=E-i 0^{+}$. This means that possible poles of $\mathcal{G}_{a, a}^{(I I)}(z)$ have a strictly negative imaginary part, as for an Hermitian FFA model. Therefore the survival probability $P(t)$ decays toward zero as $t \rightarrow \infty$ similarly to what happens in the Hermitian limit. Conversely, for an amplifying complex energy $E_{a}, \mathcal{G}_{a, a}(z)$ diverges for $z=\mathcal{E}_{0}+i 0^{+} ;$therefore $\mathcal{G}_{a, a}^{(I I)}(z)$ has a pole on the real axis, $z_{1}=\mathcal{E}_{0}$. In this case, according to Eq.(45) the survival probability $P(t)$ does not decay, and may become larger than one depending on the modulus of the residue of the dominant pole. This phenomenon is analogous to the fractional decay found in Hermitian FFA models and related to the existence of bound states (i.e. a non-empty point spectrum of $H$ ). However, in the nonHermitian FFA model with $\operatorname{Im}\left(E_{a}\right)>0$ the non-decaying behavior of $P(t)$ results from the appearance of a spectral singularity in the continuous spectrum, not from the existence of bound states. It should be noted that such a non-decaying behavior was previously predicted in the study of the lasing threshold of an optical microcavity resonantly coupled to a coupled resonator optical waveguide under special coupling conditions [35]. This unusual behavior of laser phase transition was explained as a nonmarkovian effect arising from the structured continuum of the decay channel, however it was not related to spectral singularities of the underlying Hamiltonian.

\section{SPECTRAL SINGULARITIES IN A SEMI-INFINTE TIGHT-BINDING LATTICE WITH A BOUNDARY IMPURITY SITE}

In this section we present a simple and analyticallysolvable example of a non-Hermitian FFA model showing spectral singularities, which describes rather generally single-particle electron or photon transport on a semiinfinite one-dimensional tight-binding lattice with an impurity site. The model is first presented in the framework of the general theory developed in Sec.II, and its tightbinding lattice realization is subsequently described. The physical implications of spectral singularities on wave scattering from the lattice boundary and on the decay dynamics of the impurity site are finally highlighted. 


\section{A. The Hamiltonian}

Let us consider the non-Hermitian FFA model defined by the following relations for the energy dispersion $E(k)$ and spectral coupling $v(k)$

$$
E(k)=-2 \kappa_{0} \cos k, v(k)=-\sqrt{\frac{2}{\pi}} \kappa_{a} \sin k
$$

where $\kappa_{0}, \kappa_{a}$ are two real-valued positive constants and $0 \leq k \leq \pi$. The Hermitian limit of this model, attained by assuming $\operatorname{Im}\left(E_{a}\right)=0$, is a special case of the FFA model previously investigated in Ref. 36], which is exactly solvable (see also [29]). Note that the continuous spectrum of $H$ spans the band $\left(E_{1}, E_{2}\right)$, with $E_{2}=-E_{1}=2 \kappa_{0}$. The density of states for this model is given by

$$
\rho(E)=\left(\frac{\partial E}{\partial k}\right)^{-1}=\left\{\begin{array}{cc}
\frac{1}{\sqrt{4 \kappa_{0}^{2}-E^{2}}} & -2 \kappa_{0}<E<2 \kappa_{0} \\
0 & |E|>2 \kappa_{0}
\end{array}\right.
$$

which shows van-Hove singularities at the band edges, whereas the positive spectral function $V(E)$, defined by $V(E)=\rho(E)|v(E)|^{2}$, reads

$$
V(E)=\left\{\begin{array}{cc}
\frac{\kappa_{a}^{2}}{\pi \kappa_{0}} \sqrt{1-\left(\frac{E}{2 \kappa_{0}}\right)^{2}}-2 \kappa_{0}<E<2 \kappa_{0} \\
0 & |E|>2 \kappa_{0}
\end{array}\right.
$$

which is non-singular. Substitution of Eq.(49) into Eq.(11) yields the following expression for the self-energy $\Sigma(z)$ [46]

$$
\Sigma(z)=-i \frac{\kappa_{a}^{2}}{2 \kappa_{0}^{2}}\left(\sqrt{4 \kappa_{0}^{2}-z^{2}}+i z\right)
$$

and thus [see Eq.(12)]

$$
\begin{aligned}
\Delta(\mathcal{E})= & \operatorname{Re}\left(\Sigma\left(z=\mathcal{E} \pm i 0^{+}\right)\right)= \\
= & \begin{cases}\frac{\kappa_{a}^{2}}{2 \kappa_{0}^{2}}\left(\mathcal{E}+\sqrt{\mathcal{E}^{2}-4 \kappa_{0}^{2}}\right) & \mathcal{E}<-2 \kappa_{0} \\
\frac{\kappa_{a}^{2}}{2 \kappa_{0}^{2}} \mathcal{E} & -2 \kappa_{0} \leq \mathcal{E} \leq 2 \kappa_{0} \\
\frac{\kappa_{a}^{2}}{2 \kappa_{0}^{2}}\left(\mathcal{E}-\sqrt{\mathcal{E}^{2}-4 \kappa_{0}^{2}}\right) & \mathcal{E}>2 \kappa_{0}\end{cases}
\end{aligned}
$$

The condition for the non-Hermitian Hamiltonian to possess a real-valued spectrum (i.e. to avoid complex-valued energies arising from bound states outside the continuum) is derived in Appendix B. Precisely, let $\xi_{1,2}$ be the two roots of the second-order algebraic equation

$$
\xi^{2}+\frac{E_{a}}{\kappa_{0}} \xi+1-\left(\kappa_{a} / \kappa_{0}\right)^{2}=0 .
$$

Then the Hamiltonian $H$ has a real-valued energy spectrum if and only if $\left|\xi_{1,2}\right| \leq 1$. Figure 2 shows the domain in the plane $\left(\operatorname{Im}\left(E_{a}\right) / \kappa_{0}, \kappa_{a} / \kappa_{0}\right)$ where $H$ has a purely continuous energy spectrum for a few increasing values of the ratio $\left|\operatorname{Re}\left(E_{a}\right) / \kappa_{0}\right|$. The domain lies in the sector
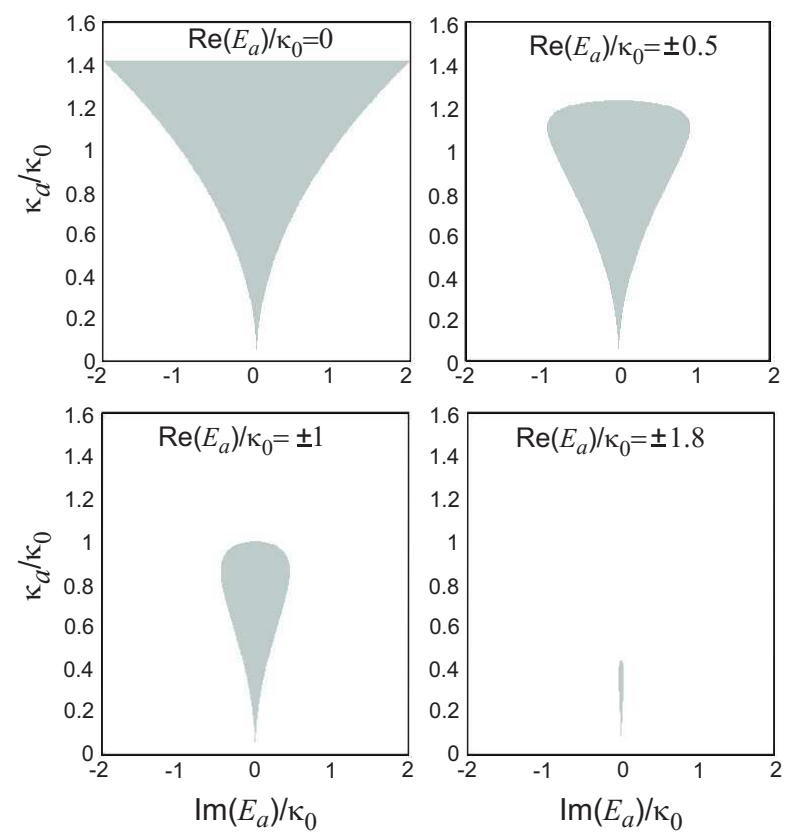

FIG. 2: Domains of non-existence of bound states for the Hamiltonian $H$ in the $\left(\operatorname{Im}\left(E_{a}\right) / \kappa_{0}, \kappa_{a} / \kappa_{0}\right)$ plane (shaded regions) for increasing values of the ratio $\left|\operatorname{Re}\left(E_{a}\right)\right| / \kappa_{0}$. For a non-Hermitian Hamiltonian, i.e. $\operatorname{Im}\left(E_{a}\right) \neq 0$, in the shaded regions the energy spectrum of $H$ is real-valued and purely continuous. Spectral singularities occur at the boundary of the shaded regions.

$\kappa_{a} / \kappa_{0} \leq \sqrt{2}$ and shrinks toward $\operatorname{Im}\left(E_{a}\right) / \kappa_{0}=\kappa_{a} / \kappa_{0}=0$ as $\left|\operatorname{Re}\left(E_{a}\right) / \kappa_{0}\right| \rightarrow 2^{-}$. For $\left|\operatorname{Re}\left(E_{a}\right) / \kappa_{0}\right| \leq 2$, bound states do exist for any value of $\kappa_{a} / \kappa_{0}$ and $\operatorname{Im}\left(E_{a}\right) / \kappa_{0}$. The wider domain is attained for $\operatorname{Re}\left(E_{a}\right)=0$. In particular, for $\operatorname{Re}\left(E_{a}\right)=0$ and $\kappa_{a} / \kappa_{0}=\sqrt{2}$, from Eq.(52) it follows that $H$ has a real-valued energy spectrum provided that

$$
-2 \kappa_{0}<\operatorname{Im}\left(E_{a}\right)<2 \kappa_{0}
$$

Let us now consider the occurrence of spectral singularities. According to Eqs.(19) and (20) and using Eqs.(49) and (51), a spectral singularity at energy $\mathcal{E}=\mathcal{E}_{0}$, inside the interval $\left(-2 \kappa_{0}, 2 \kappa_{0}\right)$, is found provided that the following two equations are simultaneously satisfied

$$
\begin{array}{r}
\operatorname{Im}\left(E_{a}\right)= \pm \frac{\kappa_{a}^{2}}{\kappa_{0}} \sqrt{1-\left(\frac{\mathcal{E}_{0}}{2 \kappa_{0}}\right)^{2}} \\
\operatorname{Re}\left(E_{a}\right)=\left(1-\frac{\kappa_{a}^{2}}{2 \kappa_{0}^{2}}\right) \mathcal{E}_{0} .
\end{array}
$$

For arbitrarily given values of $E_{a}, \kappa_{a}$ and $\kappa_{0}$, the above conditions are generally not satisfied [nowhere for $\mathcal{E}_{0}$ in the range $\left.\left(-2 \kappa_{0}, 2 \kappa_{0}\right)\right]$, i.e. the non-Hermitian FFA Hamiltonian is generally diagonalizable. Spectral singularities appear solely when a constraint among $\operatorname{Re}\left(E_{a}\right) / \kappa_{0}, \operatorname{Im}\left(E_{a}\right) / \kappa_{0}$ and $\kappa_{a} / \kappa_{0}$ is satisfied. Let us first assume $\kappa_{a} / \kappa_{0}$ strictly smaller that $\sqrt{2}$. In this case, a single spectral singularity, at the energy $\mathcal{E}_{0}=$ 


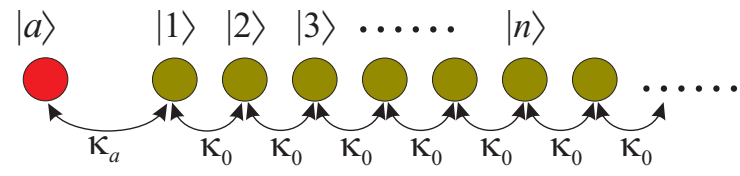

FIG. 3: (color online) Schematic of a semi-infinite onedimensional tight-binding lattice attached to a boundary impurity site $|a\rangle$.

$\operatorname{Re}\left(E_{a}\right) /\left(1-\kappa_{a}^{2} / 2 \kappa_{0}^{2}\right)$ [see Eq.(55)], is found provided that

$$
\operatorname{Im}^{2}\left(E_{a}\right)=\frac{\kappa_{a}^{4}}{\kappa_{0}^{2}}\left[1-\frac{\operatorname{Re}^{2}\left(E_{a}\right)}{\left(2 \kappa_{0}-\kappa_{a}^{2} / \kappa_{0}\right)^{2}}\right] .
$$

It can be readily shown that the condition (56) defines the boundary of the domains shown in Fig.2, i.e. a spectral singularity appears when the boundary of existence of bound states is approached. The case $\kappa_{a} / \kappa_{0}=\sqrt{2}$ is somehow singular. From Eqs.(54) and (55), for $\kappa_{a} / \kappa_{0}=$ $\sqrt{2}$ it follows that there are two spectral singularities at energies

$$
\mathcal{E}_{0}= \pm \sqrt{4 \kappa_{0}^{2}-\operatorname{Im}^{2}\left(E_{a}\right)}
$$

provided that $\operatorname{Re}\left(E_{a}\right)=0$. The physical meaning of such spectral singularities will be discussed in Sec.III.C.

\section{B. Lattice realization}

FFA models are often encountered in connection to single-particle electronic or photonic transport in onedimensional tight-binding lattices or networks (see, e.g., [25 29, 33 38] and references therein), and in most cases the underlying Hamiltonian is Hermitian. In particular, the Hermitian limit of the FFA Hamiltonian $H$ considered in the previous subsection has been previously studied in [29, 36] and shown to be equivalent to a tightbinding Hamiltonian of a semi-infinity lattice with an impurity site. The equivalence can be proven after representing the Bloch states $|k\rangle$ of the tight-binding energy band in terms of localized Wannier states $|n\rangle$ on a lattice. Let us introduce the Wannier states $|n\rangle$ as

$$
|n\rangle=\sqrt{\frac{2}{\pi}} \int_{0}^{\pi} d k \sin (n k)|k\rangle
$$

for $n=1,2,3, \ldots$. Taking into account that

$$
\int_{0}^{\pi} d k \sin (n k) \sin (m k)=\frac{\pi}{2} \delta_{n, m}
$$

$(n, m \geq 1)$, one can readily show that the Wannier states form an orthonormal system, i.e. $\langle n \mid m\rangle=\delta_{n, m}$. Additionally, from Eq.(58) it follows that the Bloch states $|k\rangle$ can be decomposed as a superposition of Wannier states $|n\rangle$ according to

$$
|k\rangle=\sqrt{\frac{2}{\pi}} \sum_{n=1}^{\infty} \sin (n k)|n\rangle .
$$

In the Wannier representation, one can readily show that

$$
\int d k E(k)|k\rangle\langle k|=-\kappa_{0} \sum_{n=1}^{\infty}(|n\rangle\langle n+1|+| n+1\rangle\langle n|)
$$

and

$$
V=\int d k\left[v(k)|a\rangle\left\langle k\left|+v^{*}(k)\right| k\right\rangle\langle a|\right]=-\kappa_{a}(|a\rangle\langle 1|+| 1\rangle\langle a|)
$$

so that the Hamiltonian $H=H_{0}+V$ can be written in the equivalent form

$$
\begin{aligned}
H= & -\kappa_{0} \sum_{n=1}^{\infty}(|n\rangle\langle n+1|+| n+1\rangle\langle n|)+E_{a}|a\rangle\langle a|+ \\
& -\kappa_{a}(|a\rangle\langle 1|+| 1\rangle\langle a|)
\end{aligned}
$$

In its present form, Eq.(63) describes single-particle electron or photon transport on a one-dimensional semiinfinite tight-binding lattice [29, 36, 37], with a hopping rate $\kappa_{0}$ between adjacent sites of the lattice and with the boundary attached to an impurity site $|a\rangle$ with 'complex' potential energy $E_{a}$ and with hopping rate $\kappa_{a}$ (see Fig.3). From a physical viewpoint, the complex potential at the boundary impurity site may account for e.g. loss of the quantum particle flux into other decay channels (quantum absorbing potentials [47]), or optical gain or loss of light waves in photonic structures [34, 35]. For instance, light transport in a semi-infinite waveguide array as in Ref. [37], but with a lossy (or active) boundary waveguide, provides a simple and experimentally accessible realization of the tight-binding model (63). It should be noted that transport and scattering phenomena in tightbinding lattices with complex potentials have been theoretically investigated in recent works (see, for instance, [47, 48]), however spectral singularities were not found in these previous models.

\section{Spectral singularities and lattice wave reflection}

The physical meaning of spectral singularities in the non-Hermitian FFA model can be captured by analyzing the wave reflection properties of the lattice of Fig.3. As it will be shown below, a spectral singularity corresponds to the appearance of a diverging peak in the reflectance spectrum when the boundary site is an 'amplifying' impurity, i.e. when $\operatorname{Im}\left(E_{a}\right)>0$, and to the vanishing of wave reflection when the boundary site is an 'absorbing' impurity, i.e. when $\operatorname{Im}\left(E_{a}\right)<0$. In the former case, we retrieve for a 'discrete' scattering problem the physical explanation of spectral singularities as resonances with vanishing spectral width, established by Mostafazadeh Ref. 9] in the framework of 'continuous' wave scattering by complex potentials. Conversely, the latter case, i.e. that of an 'absorbing' impurity site, gives a different manifestation of a spectral singularity: a wave incident on the lattice boundary is totally absorbed. 
(a)

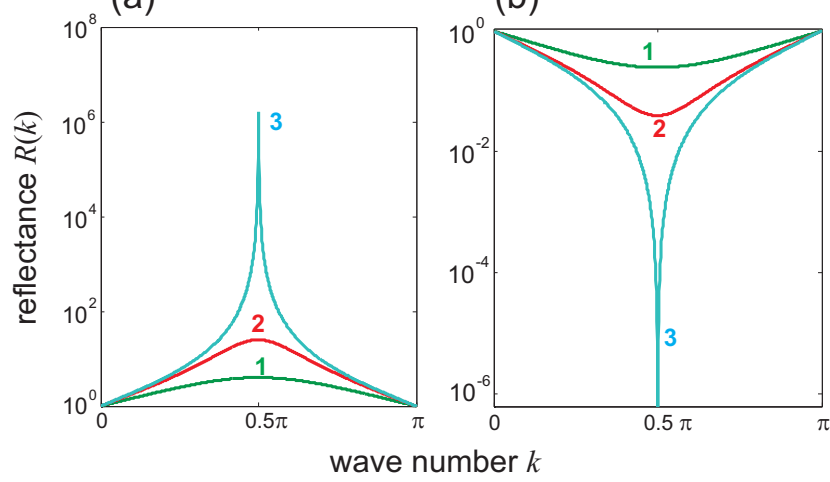

FIG. 4: (color online) Probability of particle reflection $R(k)$ for (a) an amplifying impurity site $\left[\operatorname{Im}\left(E_{a}\right)>0\right]$, and (b) an absorbing impurity site $\left[\operatorname{Im}\left(E_{a}\right)<0\right]$ for $\kappa_{a} / \kappa_{0}=1$ and $\operatorname{Re}\left(E_{a}\right)=0$. Curves 1,2 and 3 correspond to $\left|\operatorname{Im}\left(E_{a}\right)\right| / \kappa_{a}=$ $1 / 3,2 / 3$ and 1 , respectively. According to Eqs. (54) and (55), a spectral singularity occurs at $k=\pi / 2$ (i.e., $\mathcal{E}_{0}=-2 \kappa_{0} \cos k=$ 0 ) for $\left|\operatorname{Im}\left(E_{a}\right)\right| / \kappa_{a}=1$ (curve 3) (a)

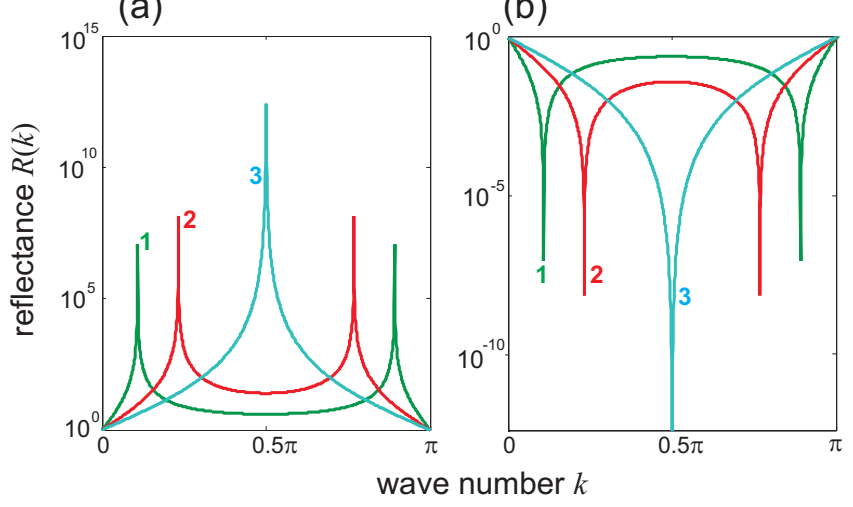

FIG. 5: (color online) Same as Fig.4, but for for $\kappa_{a} / \kappa_{0}=$ $\sqrt{2}$ and $\operatorname{Re}\left(E_{a}\right)=0$. Curves 1,2 and 3 correspond to $\left|\operatorname{Im}\left(E_{a}\right)\right| / \kappa_{a}=2 / 3,4 / 3$ and 2 , respectively. According to Eq.(57), there are two spectral singularities, corresponding to the energies $\mathcal{E}_{0}= \pm\left[4 \kappa_{0}^{2}-\operatorname{Im}^{2}\left(E_{a}\right)\right]^{1 / 2}$, which coalesce at $\left|\operatorname{Im}\left(E_{a}\right)\right| / \kappa_{a}=2$ (curve 3).
To analyze the reflection properties of the lattice shown in Fig.3, let us expand the state vector $|\psi(t)\rangle$ as

$$
|\psi(t)\rangle=c_{a}(t)|a\rangle+\sum_{n=1}^{\infty} c_{n}(t)|n\rangle
$$

where $\left|c_{n}(t)\right|^{2}$ is the occupation probability of site $|n\rangle$ and $\left|c_{a}(t)\right|^{2}$ the occupation probability of the boundary impurity site $|a\rangle$. From Eqs.(3) and (63), it follows that the occupation amplitudes $c_{n}$ and $c_{a}$ satisfy the following coupled equations

$$
\begin{aligned}
& i \frac{d c_{n}}{d t}=-\kappa_{0}\left(c_{n+1}+c_{n-1}\right) n \geq 2 \\
& i \frac{d c_{1}}{d t}=-\kappa_{0} c_{2}-\kappa_{a} c_{a} \\
& i \frac{d c_{a}}{d t}=-\kappa_{a} c_{1}+E_{a} c_{a} .
\end{aligned}
$$

Plane wave solutions to Eqs.(65-67) with wave number (momentum) $k(0 \leq k \leq \pi)$, corresponding to eigenstates of $H$ with energy $E(k)=-2 \kappa_{0} \cos k$, are of the form $c_{n}(t)=\bar{c}_{n}(k) \exp (-i E(k) t), c_{a}(t)=\bar{c}_{a}(k) \exp (-i E(k) t)$, where

$$
\begin{aligned}
& \bar{c}_{n}(k)=\exp [-i k(n-1)]+r(k) \exp [i k(n-1)] \\
& \bar{c}_{a}(k)=\frac{\kappa_{0}}{\kappa_{a}}[\exp (i k)+r \exp (-i k)]
\end{aligned}
$$

and

$$
r(k)=-\frac{\kappa_{a}^{2}-\kappa_{0}^{2}\left(2 \cos k+E_{a} / \kappa_{0}\right) \exp (i k)}{\kappa_{a}^{2}-\kappa_{0}^{2}\left(2 \cos k+E_{a} / \kappa_{0}\right) \exp (-i k)}
$$

is the spectral reflection coefficient (see, for instance, [37]). Note that $c_{n}(t)$ is given by the superposition of two traveling waves, a regressive wave $\exp [-i k n-i E(k) t]$ that propagates along decreasing values of $n$, and a progressive wave $\exp [i k n-i E(k) t]$ that propagates in the opposite direction, i.e. which is reflected from the lattice boundary [49]. The probability of reflection from the lattice boundary is given by

$$
R(k)=|r(k)|^{2}=\frac{\left[\left(\kappa_{a}^{2}-2 \kappa_{0}^{2}\right) \cos k-\kappa_{0} \operatorname{Re}\left(E_{a}\right)\right]^{2}+\left[\kappa_{a}^{2} \sin k+\kappa_{0} \operatorname{Im}\left(E_{a}\right)\right]^{2}}{\left[\left(\kappa_{a}^{2}-2 \kappa_{0}^{2}\right) \cos k-\kappa_{0} \operatorname{Re}\left(E_{a}\right)\right]^{2}+\left[\kappa_{a}^{2} \sin k-\kappa_{0} \operatorname{Im}\left(E_{a}\right)\right]^{2}}
$$

In the Hermitian $\operatorname{limit}\left(\operatorname{Im}\left(E_{a}\right)=0\right)$, one has $R(k)=1$, i.e. the incident wave is completely reflected from the lattice boundary. This is merely a consequence of conservation of the particle probability in the scattering process. Conversely, for a complex-valued energy $E_{a}$ of the impurity site, one has $R(k) \leq 1$ for $\operatorname{Im}\left(E_{a}\right)<0$, i.e. for an ab- sorbing potential, and $R(k) \geq 1$ for $\operatorname{Im}\left(E_{a}\right)>0$, i.e. for an amplifying potential. In particular, for $\operatorname{Im}\left(E_{a}\right)>0$ the reflection probability $R(k)$ goes to infinity at wave numbers $k=k_{0}$ (with $0 \leq k_{0} \leq \pi$ ) that satisfy simulta- 
neously the two conditions

$$
\begin{aligned}
\kappa_{a}^{2} \sin k_{0} & =\kappa_{0} \operatorname{Im}\left(E_{a}\right) \\
\left(\kappa_{a}^{2}-2 \kappa_{0}^{2}\right) \cos k_{0} & =\kappa_{o} \operatorname{Re}\left(E_{a}\right) .
\end{aligned}
$$

Physically, the condition $R \rightarrow \infty$ implies the existence of an outgoing wave in the lattice that is sustained by the amplifying complex potential at the impurity site. Such a divergence of $R(k)$ is the signature of a spectral singularity of $H$. In fact, taking into account that the energy of incident/reflected waves is $\mathcal{E}_{0}=-2 \kappa_{0} \cos k_{0}$, it can be easily shown that Eq.(73) is equivalent to Eq.(55), whereas Eq.(72) is equivalent to Eq.(54) with the upper (positive) sign on the right hand side. Therefore, for an amplifying impurity site $\left[\operatorname{Im}\left(E_{a}\right)>0\right]$, the condition $R \rightarrow \infty$ is equivalent to the appearance of a spectral singularity. This equivalence extends, to our scattering problem on a truncated lattice, the general result shown by Mostafazadeh [9], suggesting to interpret spectral singularities of a non-Hermitian Hamiltonian as resonances with vanishing width. However, for an absorbing potential energy at the impurity site, i.e. for $\operatorname{Im}\left(E_{a}\right)<0$, our lattice model indicates that the appearance of spectral singularities has a different physical interpretation. In fact, for $\operatorname{Im}\left(E_{a}\right)<0$ the reflection probability $R(k)$ is bounded from above and smaller than one, which prevents $R(k)$ to diverge. However, in this case $R(k)$ can vanish at wave numbers $k=k_{0}$ (with $0 \leq k_{0} \leq \pi$ ) that satisfy simultaneously the two conditions [see Eq.(71)]

$$
\begin{aligned}
\kappa_{a}^{2} \sin k_{0} & =-\kappa_{0} \operatorname{Im}\left(E_{a}\right) \\
\left(\kappa_{a}^{2}-2 \kappa_{0}^{2}\right) \cos k_{0} & =\kappa_{o} \operatorname{Re}\left(E_{a}\right) .
\end{aligned}
$$

Note that Eq.(75) is equivalent to Eq.(55), whereas Eq.(74) is equivalent to Eq.(54) with the lower (negative) sign on the right hand side. Therefore, for an absorbing impurity site $\left[\operatorname{Im}\left(E_{a}\right)<0\right]$, the appearance of a spectral singularity is equivalent to the vanishing of the reflection probability $R$ : an ingoing plane wave with wave number $k_{0}$ is fully absorbed by the impurity site at the lattice edge. An an example, Fig.4 shows the behavior of $R(k)$ for an amplifying [Fig.4(a)] and for an absorbing [Fig.4(b)] impurity site for $\operatorname{Re}\left(E_{a}\right)=0$ and $\kappa_{a} / \kappa_{0}=1$. The different curves in the figures refer to different values of $\operatorname{Im}\left(E_{a}\right) / \kappa_{0}$. Note that, at the value of $\operatorname{Im}\left(E_{a}\right) / \kappa_{0}$ corresponding to the appearance of the spectral singularity, a divergence and a zero in the $R(k)$ curve are observed in Figs.4(a) and 4(b), respectively. Figure 5 shows the behavior of $R(k)$ as in Fig.4, but for $\operatorname{Re}\left(E_{a}\right)=0$ and $\kappa_{a} / \kappa_{0}=\sqrt{2}$. In this case there are two spectral singularities at energies given by Eq.(57), which explain the two peaks $[\mathrm{Fig} .5(\mathrm{a})]$ or dips $[\mathrm{Fig} .5(\mathrm{~b})]$ in the reflectance curve $R(k)$. By increasing $\left|\operatorname{Im}\left(E_{a}\right)\right| / \kappa_{0}$, the two peaks (or dips) get closer each other, until they coalesce at $\left|\operatorname{Im}\left(E_{a}\right)\right| / \kappa_{0}=2$ (curve $3[50]$ ).

Figures 6 and 7 show two examples of wave packet reflection from the lattice boundary for an amplifying (Fig.6) and an absorbing (Fig.7) impurity site. The figures show a snapshot of $\left|c_{n}(t)\right|$ as obtained by numerical analysis of Eqs.(65-67) assuming at $t=0$ a broad
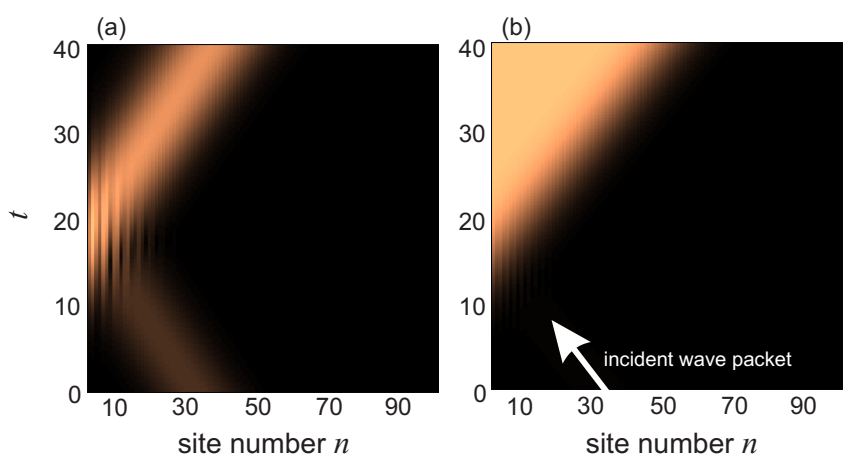

FIG. 6: (color online) Wave packet reflection in a semi-infinite lattice with an amplifying impurity site for $\kappa_{0}=\kappa_{a}=1$, $\operatorname{Re}\left(E_{a}\right)=0$ and $\operatorname{Im}\left(E_{a}\right)=1$. The initial wave packet is Gaussian- shaped, with peak amplitude $\left|c_{n}(0)\right|=1$ at $n=$ $n_{0}=30$, width $\Delta n=12$ and momentum $k=3 \pi / 10$ in (a), and $k=\pi / 2$ in (b) (corresponding to the spectral singularity energy $\left.\mathcal{E}_{0}=0\right)$. In (b)the incident wave packet, indicated by an arrow, is not visible owing to the large amplification of the reflected wave.
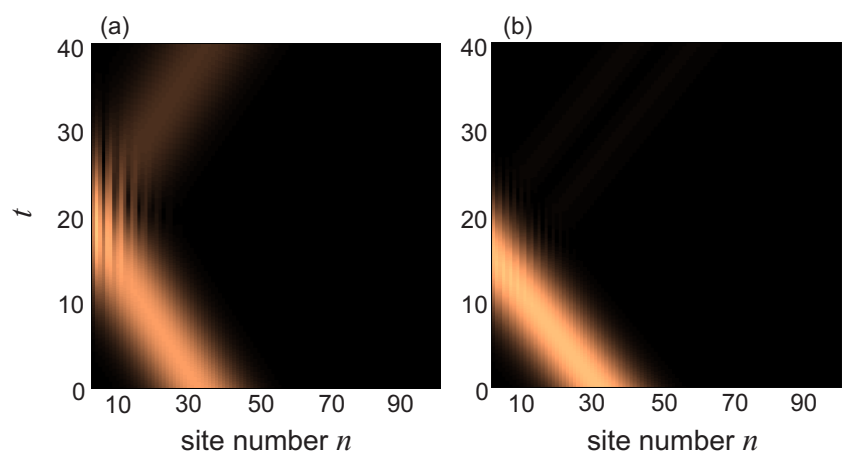

FIG. 7: (color online) Same as Fig.6, but for an absorbing impurity site $\operatorname{Im}\left(E_{a}\right)=-1$ (other parameters as in Fig.6).

Gaussian distribution of site occupation amplitudes, i.e. $c_{n}(0)=\exp \left[-\left(n-n_{0}\right)^{2} / \Delta n^{2}-i k n\right]$, where $\Delta n$ is the wave packet width, $k$ the mean wave packet momentum, and $n_{0} \gg \Delta n$ the wave packet center of mass. The large (diverging) amplification of the reflected wave packet in Fig.6(b), and the almost absence of wave packet reflection in Fig.7(b), are clearly visible when the energy $\mathcal{E}=-2 \kappa_{0} \cos k$ of the incoming wave packet attains the spectral singularity point $\mathcal{E}_{0}=0$.

The interplay between spectral singularities and decay dynamics, discussed in Sec.II.D, is exemplified in Figs.8 and 9. The system of Eqs.(65-67) has been numerically integrated with the initial condition $c_{a}(0)=1$ and $c_{n}(0)=1$. Note that, in the photonic realization of the semi-infinite lattice model of Ref. [37], such an initial condition simply corresponds to initial excitation of the boundary waveguide. The behavior of the site occupation probability $P(t)=\left|c_{a}(t)\right|^{2}$ for an amplifying and for an absorbing impurity site is depicted in Figs. 8 and 9, respectively. Note that, according to the gen- 


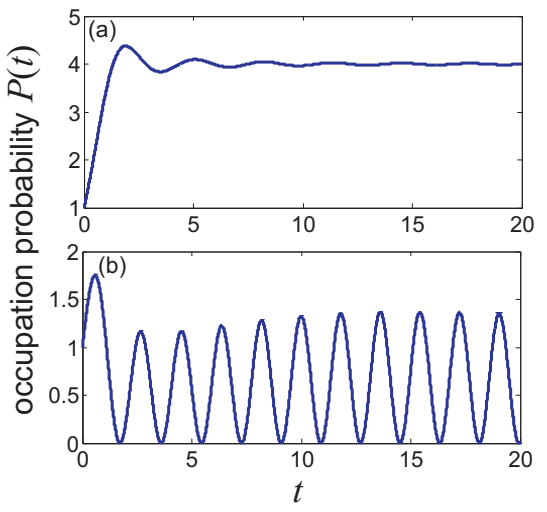

FIG. 8: (color online) Evolution of occupation probability $P(t)=\left|c_{a}(t)\right|^{2}$ in a semi-infinite lattice with an amplifying impurity site for $\operatorname{Re}\left(E_{a}\right)=0, \operatorname{Im}\left(E_{a}\right)=1$ and for (a) $\kappa_{0}=$ $\kappa_{a}=1$, (b) $\kappa_{0}=1, \kappa_{a}=\sqrt{2}$.

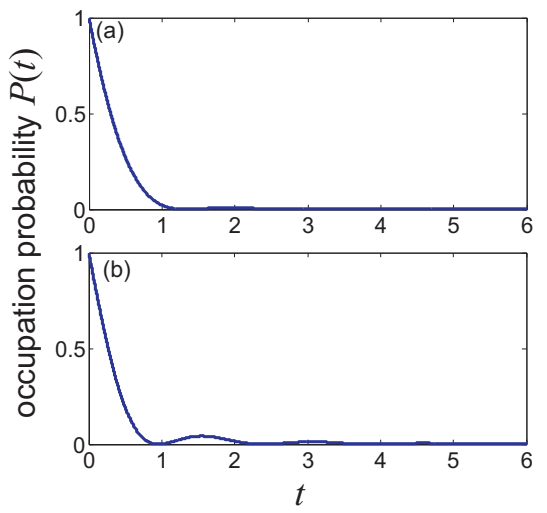

FIG. 9: (color online) Same as Fig.8, but for an absorbing impurity site $\left(\operatorname{Im}\left(E_{a}\right)=-1\right.$; other parameters as in Fig.8)

eral analysis presented in Sec.II.D, the survival probability $P(t)=\left|c_{a}(t)\right|^{2}$ decays to zero in the absorbing case (Fig.9), and the existence of a spectral singularity does not basically influence the decay dynamics of state $|a\rangle$. Conversely, for an amplifying impurity site the occupation probability $P(t)$ does not decay to zero. Note that for $\kappa_{a} / \kappa_{0}<\sqrt{2}$ [like in Fig.8(a)], $P(t)$ converges to a steady state value, whereas for $\kappa_{a} / \kappa_{0}=\sqrt{2}$ the probability $P(t)$ is an oscillating function [see Fig.8(b)]. The different behavior in the two cases is explained by observing that, for $\kappa_{a} / \kappa_{0} \neq \sqrt{2}$ there is one spectral singularity and thus in Eq.(45) there is only one pole that contributes to the non-decaying part of $c_{a}(t)$. On the other hand, for the somehow special case $\kappa_{a} / \kappa_{0}=\sqrt{2}$ the Hamiltonian $H$ has two spectral singularities [see Eq.(57)], and in Eq.(45) there are two poles that contribute to the non-decaying part of $c_{a}(t)$. The interference of these two non-decaying terms explains the oscillatory behavior of $P(t)$ in Fig.8(b) (see also [35]).

\section{CONCLUSIONS}

In this work a comprehensive analysis of the spectral properties of a non-Hermitian extension of the FriedrichsFano-Anderson model has been presented. The FFA model generally describes the decay of a discrete state $|a\rangle$ of energy $E_{a}$ coupled to a continuum of states. Here we have extended the ordinary model by allowing the energy $E_{a}$ to become complex-valued, with either $\operatorname{Im}\left(E_{a}\right)>0$ (the 'amplifying' case) or $\operatorname{Im}\left(E_{a}\right)<0$ (the 'absorbing' case). Contrary to the Hermitian FFA, it has been shown by a direct diagonalization procedure and by the analysis of the resolvent operator that spectral singularities in the continuous spectrum may exist for both the amplifying and the absorbing non-Hermitian FFA model. The physical implications and relevance of spectral singularities have been discussed, in particular with reference to a tight-binding realization of the non-Hermitian FFA model that describes single-particle electronic or photonic transport in a semi-infinite lattice attached to an impurity site with a complex energy. Different behaviors have been found for an amplifying and for an absorbing impurity site, reflecting the circumstance that the divergence of the resolvent $G(z)$ appears when the spectral singularity is approached either from above or from below of the complex energy plane. For an amplifying impurity site, the spectral singularity manifests itself as a divergence of the reflection probability from the lattice boundary, a result which is the 'discrete' analogous of the general result recently established by Mostafazadeh for wave scattering by complex potentials in the framework of the continuous Schrödinger equation 11]. As compared to Ref. 11], here we have also clarified the physical relevance of spectral singularities in the temporal domain, showing that in the amplifying non-Hermitian FFA Hamiltonian the spectral singularity of the resolvent prevents the decay of state $|a\rangle$ into the continuum, in spite of the absence of bound states. For an absorbing impurity site, we have shown that a spectral singularity corresponds to a zero of the reflection probability from the lattice boundary. This result is clearly not observable in the problem of wave scattering from complex barriers addressed in Refs. 11, 12], where the double-degeneracy of energy levels plays a major role and a spectral singularity can never correspond to the total absorption of the incident wave.

Owing to the importance of the FFA model in different areas of physics, it is envisaged that the present analysis may stimulate further theoretical and experimental studies aimed to investigate the unique features of nonHermitian physical systems. In particular, recent results obtained in photonic systems based on coupled waveguides or arrays of coupled waveguides with controlled regions of optical gain and/or loss [51], indicate that engineered photonic systems might provide an accessible laboratory tool to experimentally observe spectral singularities. 


\section{Appendix A: Matrix elements of the resolvent}

In this Appendix we derive the expressions of the matrix elements $\mathcal{G}_{a, a}(z)=\langle a \mid G(z) a\rangle, \mathcal{G}_{k, a}(z)=\langle k \mid G(z) a\rangle$, $\mathcal{G}_{a, k}(z)=\langle a \mid G(z) k\rangle$ and $\mathcal{G}_{k, k^{\prime}}(z)=\left\langle k \mid G(z) k^{\prime}\right\rangle$ of the resolvent [Eqs.(6-9) given in the text].

From the identity $G(z)\left(z-H_{0}-V\right)=\left(z-H_{0}-V\right) G(z)=$ $\mathcal{I}$ it follows that

$$
\begin{aligned}
\left\langle a \mid G(z)\left(z-H_{0}\right) a\right\rangle-\langle a \mid G(z) V a\rangle & =1 \\
\left\langle a \mid G(z)\left(z-H_{0}\right) k\right\rangle-\langle a \mid G(z) V k\rangle & =0 \\
\left\langle k \mid\left(z-H_{0}\right) G(z) a\right\rangle-\langle k \mid V G(z) a\rangle & =0 \\
\left\langle k \mid G(z)\left(z-H_{0}\right) k^{\prime}\right\rangle-\left\langle k \mid G(z) V k^{\prime}\right\rangle & =\delta\left(k-k^{\prime}\right)
\end{aligned}
$$

Taking into account that

$$
V|a\rangle=\int d k v^{*}(k)|k\rangle, V|k\rangle=v(k)|a\rangle
$$

and that $\left(z-H_{0}\right)|a\rangle=\left(z-E_{a}\right)|a\rangle,\left(z-H_{0}\right)|k\rangle=(z-$ $E(k))|k\rangle$, Eqs.(A1) and (A2) take the form

$$
\begin{array}{r}
\left(z-E_{a}\right) \mathcal{G}_{a, a}(z)-\int d k v^{*}(k) \mathcal{G}_{a, k}=1 \\
(z-E(k)) \mathcal{G}_{a, k}(z)-v(k) \mathcal{G}_{a, a}(z)=0
\end{array}
$$

which can be solved for $\mathcal{G}_{a, a}$ and $\mathcal{G}_{a, k}$, yielding Eqs.(6) and (7) given in the text. To calculate $\mathcal{G}_{k, a}(z)$, we use Eq.(A3) and note that $\left\langle k \mid\left(z-H_{0}\right) G(z) a\right\rangle=\left\langle\left(z^{*}-\right.\right.$ $\left.H_{0}^{\dagger}\right) k|G(z) a\rangle=(z-E(k)) \mathcal{G}_{k, a}(z)$ and $\langle k \mid V G(z) a\rangle=$ $\langle V k \mid G(z) a\rangle=v^{*}(k) \mathcal{G}_{a, a}(z)$. This yields $(z-$ $E(k)) \mathcal{G}_{k, a}(z)-v^{*}(k) \mathcal{G}_{a, a}(z)=0$, which can be solved for $\mathcal{G}_{k, a}(z)$, yielding Eq.(8) given in the text. Finally, the matrix element $\mathcal{G}_{k, k^{\prime}}(z)$ is obtained from Eq.(A4), which can be written in the form $\left(z-E\left(k^{\prime}\right)\right) \mathcal{G}_{k, k^{\prime}}(z)-v\left(k^{\prime}\right) \mathcal{G}_{k, a}(z)=$ $\delta\left(k-k^{\prime}\right)$, i.e.

$$
\mathcal{G}_{k, k^{\prime}}(z)=\frac{v\left(k^{\prime}\right) \mathcal{G}_{k, a}(z)}{z-E\left(k^{\prime}\right)}+\frac{\delta\left(k-k^{\prime}\right)}{z-E\left(k^{\prime}\right)} .
$$

Substitution of Eq.(8) into Eq.(A8) finally yields Eq.(9) given in the text.

\section{Appendix B: Conditions for a real-valued energy spectrum of the non-Hermitian Hamiltonian}

In this Appendix we derive the necessary and sufficient conditions that ensure a real-valued energy spectrum for the non-Hermitian FFA Hamiltonian $H$ introduced in Sec.III.A. As shown in Sec.II.B, this condition is equivalent to the vanishing of the point-spectrum of $H$, i.e. to the absence of bound states. The detailed calculations can be performed following two different, though equivalent, approaches. The first one starts from the representation of $H$ in the $\{|a\rangle,|k\rangle\}$ basis (the Bloch basis), whereas the second approach uses a different decomposition of $H$, namely on the $\{|a\rangle,|n\rangle\}$ basis, where $|n\rangle$ are the Wannier states introduced in Sec.III.B (the Wannier basis). For the sake of completeness, we present the detailed calculations for both approaches.

1. Bloch-basis representation of $H$. As shown in Sec.II.B, the absence of bound states of $H$ requires that Eq.(18) does not admit of any solution in the complex $z$ plane. Using the expression (50) of the self-energy $\Sigma(z)$, Eq.(18) takes the form

$$
\left(1-\frac{\kappa_{a}^{2}}{2 \kappa_{0}^{2}}\right) z-E_{a}=-i \frac{\kappa_{a}^{2}}{2 \kappa_{0}^{2}} \sqrt{4 \kappa_{0}^{2}-z^{2}} .
$$

We can solve Eq.(B1) by introducing, in place of $z$, the new complex-valued variable $\mu$ defined by

$$
z=-\kappa_{0}[\exp (\mu)+\exp (-\mu)]=-2 \kappa_{0} \cosh \mu .
$$

Without loss of generality, we may assume $\operatorname{Re}(\mu)>0$. In fact, the function $z(\mu)$ defined by Eq.B(2) is invariant for the inversion $\mu \rightarrow-\mu$, so that we may restrict our analysis to the case $\operatorname{Re}(\mu)>0$. With such a substitution, the square root on the right hand side of Eq.(B1) can be solved analytically, yielding $\pm 2 i \kappa_{0} \sinh \mu$. Some care should be taken when choosing the right determination (i.e. sign) of the square root [46]. For $\operatorname{Re}(\mu)>0$, one obtains

$$
2\left(1-\frac{\kappa_{a}^{2}}{2 \kappa_{0}^{2}}\right) \cosh \mu+\frac{E_{a}}{\kappa_{0}}=-\frac{\kappa_{a}^{2}}{\kappa_{0}^{2}} \sinh \mu .
$$

After setting $\xi=\exp (\mu)$, from Eq.(B3) one obtains Eq.(52) given in the text once $\cosh \mu$ and $\sinh \mu$ are expressed in terms of the exponentials $\exp ( \pm \mu)=\xi^{ \pm 1}$. Therefore, if the two roots $\xi_{1,2}$ of Eq.(52) satisfy the condition $\left|\xi_{1,2}\right| \leq 1$, Eq.(B3) does not have roots with $\operatorname{Re}(\mu)>0$, and hence $H$ does not have bound states.

2. Wannier-basis representation of $H$. In this approach, we use the tight-binding representation of the Hamiltonian $H$ using the Wannier function basis [Eq.(63)]. Bound states of $H$ correspond in this case to surface states localized near the edge of the truncated lattice of Fig.3. They can be directly determined by looking for a solution to Eqs.(65-67) of the form

$$
c_{n}(t)=\exp [-\mu(n-1)-i E t], c_{a}(t)=A \exp (-i E t)
$$

$(n \geq 1)$, where $E$ is the energy of the surface state. The constants $\mu$ and $A$, as well as the dependence of $E$ on $\mu$, are readily determined by substituting Eq.(B4) into Eqs.(65-67). One obtains

$$
\begin{aligned}
E & =-2 \kappa_{0} \cosh \mu \\
E & =-\kappa_{0} \exp (-\mu)-\kappa_{a} A \\
E A & =-\kappa_{a}+E_{a} A .
\end{aligned}
$$

from which the following second-order algebraic equations for $\xi=\exp (\mu)$ is readily obtained

$$
\xi^{2}+\frac{E_{a}}{\kappa_{0}} \xi+1-\frac{\kappa_{a}^{2}}{\kappa_{0}^{2}}=0
$$


which is Eq.(52) given in the text. Localization of the surface state at the lattice edge requires $c_{n} \rightarrow 0$ as $n \rightarrow$ $\infty$, i.e. $\operatorname{Re}(\mu)>0$ [see Eq.(B4)]. Therefore, if the two roots $\xi_{1,2}$ of Eq.(B8) satisfy the condition $\left|\xi_{1,2}\right| \leq 1$, there are not surface states at the lattice edge.
[1] C.M. Bender, Rep. Prog. Phys. 70, 947 (2007).

[2] P. Dorey, C. Dunning, and R. Tateo, J. Phys.A 40, R205 (2007).

[3] A. Mostafazadeh, "Pseudo-Hermitian Quantum Mechanics", arXiv:0810.5643v2 (2008).

[4] C.M. Bender and S. Boettcher, Phys. Rev. Lett. 80, 5243 (1998).

[5] T. Kato, Perturbation Theroy of Linear Operators (Springer, Berlin, 1966).

[6] M.V. Berry, Czech. J. Phys. 54, 1039 (2004).

[7] R. R.D. Kemp, Can. J. Math. 10, 447 (1958); M. A. Naimark, Amer. Math. Soc. Transl. 16, 103 (1960); J. Schwartz, Commun. Pure Appl. Math. 13, 609 (1960); V. E. Ljance, Amer. Math. Soc. Trans. 60, 185 (1967).

[8] B. F. Samsonov, J. Phys. A 38, L397 (2005).

[9] A. Mostafazadeh and H. Mehri-Dehnavi, J. Phys. A 42, 125303 (2009).

[10] W. D. Heiss, Phys. Rep. 242, 443 (1994); E. Narevicius and N. Moiseyev, Phys. Rev. Lett. 81, 2221 (1998); C. Dembowski, H.-D. Gräf, H. L. Harney, A. Heine, W. D. Heiss, H. Rehfeld, and A. Richter, Phys. Rev. Lett. 86, 787 (2001); C. Dembowski, B. Dietz, H.-D. Graf, H. L. Harney, A. Heine, W. D. Heiss, and A. Richter, Phys. Rev. E 69, 056216 (2004); T. Stehmann, W. D. Heiss, and F. G. Scholtz, J. Phys. A 37, 7813 (2004); J. Rubinstein, P. Sternberg, and Q. Ma, Phys. Rev. Lett. 99, 167003 (2007); U. Günther, I. Rotter, and B. Samsonov, J. Phys. A 40, 8815 (2007); P. Cejnar, S. Heinze, and M. Macek, Phys. Rev. Lett. 99, 100601 (2007); S. Klaiman, U. Günther, and N. Moiseyev, Phys. Rev. Lett. 101, 080402 (2008); M. Müller and I. Rotter, J. Phys. A 41, 244018 (2008).

[11] A. Mostafazadeh, Phys. Rev. Lett. 102, 220402 (2009).

[12] A. Mostafazadeh, J. Phys. A 39, 13495 (2006).

[13] Z. Ahmedar, "Zero width resonance (spectral singularity) in a complex PT-symmetric potential", Xiv:0908.2876v1 (2009).

[14] J.G. Muga, J.P. Palao, B. Navarro, and I.L. Egusquiza, Phys. Rep. 395, 357 (2004).

[15] A. Mostafazadeh, "Resonance Phenomenon Related to Spectral Singularities, Complex Barrier Potential, and Resonating Waveguides", arXiv:0908.1713v1 (2009).

[16] A. Ruschhaupt, F. Delgado, and J.G. Muga, J. Phys. A 38, L171 (2005).

[17] K.O. Friedrichs, Commun. Pure Appl. Math. 1, 361 (1948).

[18] U. Fano, Phys. Rev. 124, 1866 (1961).

[19] P.W. Anderson, Phys. Rev. 164, 41 (1961).

[20] B. Piraux, R. Bhatt, and P.L. Knight, Phys. Rev. A 41, 6296 (1990).

[21] P.L. Knight, M.A. Lauder, and B.J. Dalton, Phys. Rep. 190, 1 (1990).

[22] C. Cohen-Tannoudji, J. Dupont-Roc, and G. Grynberg, Atom-Photon Interactions (Wiley, New York, 1992).

[23] A.G. Kofman, G. Kurizki, and B. Sherman, J. Mod. Opt. 41, 353 (1994).
[24] P. Lambropoulos, G.M. Nikolopoulos, T.R. Nielsen, and S. Bay, Rep. Prog. Phys. 63, 455 (2000).

[25] G.D. Mahan, Many-Particle Physics (New York, Plenum Press, 1990), pp.272-285.

[26] M. Cini, Topics and Methods in Condended-Matter Theory (Springer, Heidelberg, 2007), Chap.5, pp.81-89.

[27] J.W. Gadzuk and M. Plihal, Farady Discuss. 117, 1 (2000).

[28] N. Stefanou and A. Modinos, Phys. Rev. B 57, 12127 (1998); P. A. Orellana, M. L. Ladrón de Guevara, and F. Claro, Phys. Rev. B 70, 233315 (2004); L. Zhou, F.M. Hu, J. Lu, and C.P. Sun, Phys. Rev. A 74, 032102 (2006); E. Rufeil Fiori and H.M. Pastawski, Chem. Phys. Lett. 420, 35 (2006); G.-B. Zhang, S.-J. Wang, and L. Li, Phys. Rev. B 74, 085106 (2006); A. V. Malyshev, P. A. Orellana, and F. Domínguez-Adame, Phys. Rev. B 74, 033308 (2006); P. Zedler, G. Schaller, G. Kiesslich, C. Emary, and T. Brandes, Phys. Rev. B 80, 045309 (2009).

[29] S. Tanaka, S. Garmon, and T. Petrosky, Phys. Rev. B 73, 115340 (2006); S. Tanaka, S. Garmon, G. Ordonez, and T. Petrosky, Phys. Rev. B 76, 153308 (2007); H. Nakamura, N. Hatano, S. Garmon, and T. Petrosky, Phys. Rev. Lett 99, 210404 (2007).

[30] T. Petrosky, I. Prigogine and S. Tasaki, Physica A 173, 175 (1991); G. Ordonez, T. Petrosky, and I. Prigogine, Phys. Rev. A 63, 052106 (2001).

[31] H. Nakazato, M. Namiki, and S. Pascazio, Int. J. Mod. Phys. B 10, 247 (1996); P. Facchi, H. Nakazato, and S. Pascazio, Phys. Rev. Lett. 86, 2699 (2001); P. Facchi and S. Pascazio, La Regola dOro di Fermi, in Quaderni di Fisica Teorica, edited by S. Boffi (Bibliopolis, Napoli, 1999).

[32] A.G. Kofman and G. Kurizki, Nature (London) 405, 546 (2000); A.G. Kofman and G. Kurizki, Phys. Rev. Lett. 87, 270405 (2001); X.-H. Wang, B.-Y. Gu, R. Wang, and H.-Q. Xu, Phys. Rev. Lett.91, 113904 (2003); J. Martorell, D.W.L. Sprung, W. van Dijk, and J.G. Muga, Phys. Rev. A 79, 062104 (2009).

[33] S. Fan, P. R. Villeneuve, J. D. Joannopoulos, and H. A. Haus, Phys. Rev. Lett. 80, 960 (1998); S. Fan, P. R. Villeneuve, J. D. Joannopoulos, M. J. Khan, C. Manolatou, and H. A. Haus, Phys. Rev. B 59, 15882 (1999); P. Chak, S. Pereira, and J. E. Sipe, Phys. Rev. B 73, 035105 (2006); L.-L. Lin, Z.-Y. Li, and B. Lin, Phys. Rev. B 72, 165330 (2005).

[34] Y. Xu, Y. Li, R. K. Lee, and A. Yariv, Phys. Rev. E 62, 7389 (2000).

[35] S. Longhi, Phys. Rev. A 74, 063826 (2006).

[36] S. Longhi, Eur. Phys. J. B 57, 45 (2007).

[37] S. Longhi, Phys. Rev. Lett. 97, 110402 (2006); P. Biagoni, G. Della Valle, M. Ornigotti, M. Finazzi, L. Duo, P. Laporta and S. Longhi, Optics Express 16, 3762 (2008).

[38] A.E. Miroshnichenko, S. Flach, and Y.S. Kivshar, "Fano resonances in nanoscale structures", arXiv:0902.3014 4 (2009).

[39] G. Sudarshan, in Field Theory, Quantization and Statis- 
tical Physics, edited by E. Tirapegui (Reidel, Dordrecht, 1981), pp. 237-245.

[40] M. Miyamoto, Phys. Rev. A 72, 063405 (2005).

[41] For $\operatorname{Im}\left(E_{a}\right)=0$, we recover the Hermitian limit of the FFA Hamiltonian. In this case a divergence of the resolvent for $z \rightarrow \mathcal{E}_{0}$, obtained from Eq.(20) with $V\left(\mathcal{E}_{0}\right)=0$, corresponds to a bound state embedded into the continuum (see, e.g., [39, 40]) rather than to a spectral singularity of $H$.

[42] In fact, if $z$ were a real-valued root of Eq.(18) outside the interval $\left(E_{1}, E_{2}\right)$, according to Eq.(12) one would have $\Sigma(z)=\Delta(z)$ because $V(z)=0$. Hence, $\Sigma(z)$ turns out to be real-valued. To satisfy Eq.(18), the condition $\operatorname{Im}\left(E_{a}\right)=0$ must be thus satisfied. This means that a real-valued root $z$ of Eq.(18) necessarily requires $H$ to be Hermitian.

[43] Since the divergence of $G(z)$ at $z=\mathcal{E}_{0}$ on the continuous spectrum occurs when $z$ approaches $\mathcal{E}_{0}$ solely from one side (either from the top or from the bottom) of the complex plane, $\mathcal{E}_{0}$ does not belong to the point spectrum of $H$, rather it is a spectral singularity. In fact, if $\mathcal{E}_{0}$ were an eigenvalue of $H$ corresponding to a square-integrable eigenfunction $\left|\mathcal{E}_{0}\right\rangle$, denoting by $\left|\mathcal{E}_{0}^{\dagger}\right\rangle$ the eigenfunction of the adjoint $H^{\dagger}$ corresponding to the same eigenvalue and taking in Eq.(5) $|\chi\rangle=\left|\mathcal{E}_{0}^{\dagger}\right\rangle$ and $|\varphi\rangle=\left|\mathcal{E}_{0}\right\rangle$, one would obtain $\mathcal{G}_{\chi, \varphi}(z)=\left\langle\mathcal{E}_{0}^{\dagger} \mid \mathcal{E}_{0}\right\rangle /\left(z-\mathcal{E}_{0}\right)$. Since $\left\langle\mathcal{E}_{0}^{\dagger} \mid \mathcal{E}_{0}\right\rangle$ is finite, it would then follow that $\mathcal{G}_{\chi, \varphi}(z)$ should diverge when both $z=\mathcal{E}_{0}+i 0^{+}$and $z=\mathcal{E}_{0}-i 0^{+}$.

[44] For a featureless continuum, for which $\Delta(\mathcal{E})$ and $V(\mathcal{E})$ are smooth functions of $\mathcal{E}$, the resonance curve (39) has a Lorentzian shape and the quantum system, intially prepared in state $|a\rangle$, would decay into the continuum following a (nearly) exponential decay law with lifetime $1 /\left[\pi V\left(E_{a}\right)\right]$ (Weisskopf-Wigner or Breit-Wigner approx- imation). See also the discussion in Sec.II.D.

[45] We assume here that the spectrum of $H$ is purely continuous, i.e. we assume that there are not bound states (outside or embedded in the continuum). In this case, for the Hermitian FFA model the decay of the survival probability $P(t)$ is always complete.

[46] The proper determination of the square root on the right hand side of Eq.(50) must be chosen such that $\operatorname{Im}\left(\Sigma\left(z=\mathcal{E} \pm i 0^{+}\right)\right)=\mp \pi V(\mathcal{E})$, according to Eq.(12).

[47] F. Dǒgan, W. Kim, C.M. Blois, and F. Marsiglio, Phys. Rev. B 77, 195107 (2008).

[48] O. Bendix, R. Fleischmann, T. Kottos, and B. Shapiro, Phys. Rev. Lett. 103, 030402 (2009).

[49] More precisely, far from the lattice boundary a wave packet of the form $c_{n}(t)=\int d k Q(k) \exp [-i k n-i E(k) t]$, with spectrum $Q(k)$ narrow at around $k=k_{0}(0<$ $\left.k_{0}<\pi\right)$, propagates along the lattice with a group velocity $v_{g}=d n / d t=-2 \kappa_{0} \sin k_{0}<0$, i.e. the wave packet is incident onto the lattice boundary. Conversely, a wave packet formed by the superposition $c_{n}(t)=$ $\int d k Q(k) \exp [i k n-i E(k) t]$, with spectrum $Q(k)$ narrow at around $k=k_{0}$, propagates along the lattice with a group velocity $v_{g}=2 \kappa_{0} \sin k_{0}>0$, i.e. it is reflected from the lattice edge.

[50] At the coalescent point, the resolvent $G(z)$ has a secondorder pole at $z=0$.

[51] R. El-Ganainy, K. G. Makris, D. N. Christodoulides, and Z. H. Musslimani, Opt. Lett. 32, 2632 (2007); K.G. Makris, R. El-Ganainy, D.N. Christodoulides, and Z.H. Musslimani, Phys. Rev. Lett. 100, 103904 (2008); A. Guo, G.J. Salamo, D. Duchesne, R. Morandotti, M. Volatier-Ravat, V. Aimez, G. A. Siviloglou, and D. N. Christodoulides, Phys. Rev. Lett. 103, 093902 (2009). 